DOI: $10.1002 /(($ please add manuscript number $))$

Article type: Communication

\title{
Sub-3 nm Ultrafine Monolayer Layered Double Hydroxide Nanosheets for Electrochemical Water Oxidation
}

Yufei Zhao, Xin Zhang, Xiaodan Jia, Geoffrey I.N. Waterhouse, Run Shi, Xuerui Zhang, Fei Zhan, Ye Tao, Li-Zhu Wu, Chen-Ho Tung, Dermot O'Hare and Tierui Zhang*

Dr. Y. Zhao, X. Zhang, R. Shi, X. R. Zhang, Prof. L.-Z. Wu, Prof. C.-H. Tung, Prof. T. Zhang

Key Laboratory of Photochemical Conversion and Optoelectronic Materials, Technical Institute of Physics and Chemistry, Chinese Academy of Sciences, Beijing 100190, China

Tel: +8610 82543428; Fax: +861062554670

E-mail: tierui@mail.ipc.ac.cn

X. Zhang, R. Shi, X. R. Zhang

University of Chinese Academy of Sciences, Beijing 100049, P. R. China

Dr. X. Jia

College of Chemistry and Chemical Engineering, Xi'an University of Science and Technology, Xi'an 710054, Shanxi, P. R. China

Prof. G. I.N. Waterhouse

School of Chemical Sciences, The University of Auckland, Auckland 1142, New Zealand

Dr. F. Zhan, Prof. Y. Tao

Institute of High Energy Physics, Chinese Academy of Sciences, Beijing 100049, P. R.

China

Prof. D. O’Hare

Chemistry Research Laboratory, Department of Chemistry, University of Oxford, Mansfield Road, Oxford OX1 3TA, United Kingdom

Y. Zhao, X. Zhang contributed equally to this work.

Keywords: layered double hydroxide; monolayer nanosheet; defect; water oxidation 
Abstract: We report the synthesis of ultrafine NiFe-layered double hydroxide (NiFeLDH) nanosheets, possessing a mean lateral size of less than $3 \mathrm{~nm}$ with thickness of 0.6 $\mathrm{nm}$. Abundant cation and oxygen vacancies impart these ultrafine nanosheets with semi-metallic character, and thus superior charge transfer properties and electrochemical water oxidation performance with overpotentials $(\eta)$ of $254 \mathrm{mV}$ relative to monolayer LDH nanosheets ( $\eta$ of $280 \mathrm{mV}$ ) or bulk LDH materials ( $\eta$ of 320 $\mathrm{mV})$. These results are highly encouraging for the future application of ultrafine monolayer LDH nanosheets in electronics, solar cells and catalysis.

Electrocatalytic water splitting involving oxygen evolution reaction (OER) and hydrogen evolution reaction (HER) is a promising technology for generating hydrogen from water, and widely considered a cornerstone technology in the development of future hydrogen economies. ${ }^{[1]}$ The overall efficiency of the electrocatalytic water splitting process is generally limited by slow kinetics of OER, a complex four-electron redox process involving $\mathrm{O}-\mathrm{O}$ bond formation that typically requires a high overpotential. $\mathrm{RuO}_{2}$ and $\mathrm{IrO}_{2}$ are currently the state-of-the-art materials for OER, though the high cost and low earth abundance of Ru and Ir motivates the search for low cost alternatives. Layered double hydroxide $(\mathrm{LDH})$, due to the flexible chemical composition, show great potential in photo/electrocatalysis. ${ }^{[2]}$ Since the first report of NiFe-LDH-based materials exhibiting high OER activity, ${ }^{[3]} \mathrm{Hu}$ and co-workers have reported that monolayer NiM-LDH nanosheets $(\mathrm{M}=\mathrm{Fe}, \mathrm{Co}$, etc. $)$ exhibit efficient performance for water oxidation at low overpotentials $\left(0.3 \mathrm{~V}\right.$ at $\left.10 \mathrm{~mA} \mathrm{~m}^{-2}\right) \cdot{ }^{[4]} \mathrm{CoMn},{ }^{[5]}$ $\mathrm{NiCo},{ }^{[6]} \mathrm{NiV},{ }^{[7]} \mathrm{VFe}$-based ultrathin LDH nanosheets also show good performance in OER, mainly due to the high surface area and the exposure of active surface sites. ${ }^{[8]}$ However, the LDH nanosheet catalysts reported to date generally possess lateral platelet dimensions above $30 \mathrm{~nm}$ (Table S1), these platelets are too large to dramatically improve the catalytic performance due to the limited availability of edge and corner sites which are typically highly reactive sites due to coordinative unsaturation. ${ }^{[9]}$ Although the catalytic activity can be partly improved by loading LDH nanosheet on a 
substrate to increase the quantity of these reactive interfaces, it is still highly challenging to obtain ultrafine monolayer LDH with the lateral size of less than $3 \mathrm{~nm}$ containing highly exposed coordinatively unsaturated edge or corner active sites.

Transitionally, the size of LDHs nanosheets can be successfully controlled within lateral size of 30-200 $\mathrm{nm}$ and monolayer thicknesses by using top down (including solvent exfoliation ${ }^{[10]}$ and plasma etching ${ }^{[11]}$ ) or bottom up approaches ${ }^{[4,}$ 13a, 14] (microemulsion methods, ${ }^{[12]}$ layer growth inhibitors ${ }^{[13]}$ ).. Reducing the lateral size further to sub-3 $\mathrm{nm}$ is challenging due to rapid crystallization kinetics and/or platelet aggregation. ${ }^{[15]}$ Recently, $7.8 \mathrm{~nm}$ LDHs nanoclusters containing only several layers were obtained using propylene oxide and acetylacetone as solvents, ${ }^{[16]}$ and some $\sim 5 \mathrm{~nm}$ LDH nanosheets have been reported when LDHs were grown in-situ on graphene-based supports. ${ }^{[17]}$ Such approaches to ultrafine LDH nanosheets have limited practical value due to the low production yield. The discovery of a simple and facile route to monolayer LDH nanodots with lateral size less than $3 \mathrm{~nm}$ that should intrinsically possess an abundance of the key surface sites is the motivation for this current work. The aim is that such engineered nanomaterials should demonstrate excellent OER performance. To our knowledge, no prior work has been reported on the successfully synthesis of support-free sub-3 nm monolayer LDHs.

Recently, significant effort has been directed towards the preparation of novel ultrafine nanosheets materials, ${ }^{[18]}$ by using top-down (sonication method) or bottom up methods. The successful synthesis of ultrafine nanosheets has been reported in chargeneutral layered materials (graphene, ${ }^{[19]}$ carbon, ${ }^{[20]}$ phosphorene, ${ }^{[21]}$ hexagonal $\mathrm{BN}$ and transition-metal dichalcogenides (TMDs) ${ }^{[22]}$ ) and charge-negative materials (carbon nitride,${ }^{[23]}$ transition metal oxides).$^{[9,24]}$ These materials display unprecedented physical, electronic and chemical properties, and they are finding new applications in sensing, electronics, catalysis, supercapacitors and batteries, amongst others. ${ }^{[25]}$ This inspired us to explore the synthesis of ultrafine positively charged LDH nanosheets for catalytic applications.

Herein, we report the synthesis of ultrafine monolayer NiFe-LDH nanosheets (size 
$2.3 \mathrm{~nm}$, thickness $0.6 \mathrm{~nm}$ with a narrow platelet size distributions, denoted as LDH-UF), we show that these materials display higher OER activity than monolayer NiFe-LDH nanosheets. The LDH-UF samples were obtained in high yield by the pulsed ultrasonication of monolayer LDH nanosheet precursors in formamide (Scheme 1). Extended X-ray absorption fine structure (EXAFS) studies and density functional theory (DFT) calculations revealed that the LDH-UF possess a higher concentration of cation and anion (i.e. oxygen) vacancies compared with the monolayer NiFe-LDH nanosheet precursor or bulk NiFe-LDH, which gave the LDH-UF semi-metallic character. The high conductivity of LDH-UF, along with an abundance of exposed surface sites (especially sheet edges), resulted in the nanodots displaying excellent performance for electrocatalytic water oxidation compared with monolayer LDH nanosheets.

To synthesize LDH-UF, we used monolayer LDH nanosheets as the precursor. Generally such monolayer sheets are obtained by top-down methods involving the exfoliation of bulk LDH in highly polar solvents such as formamide, though such processes typically take up to 1-3 days to yield LDH monolayers. ${ }^{[10]}$ The monolayer LDH nanosheets used in this study were prepared by a novel process wherein formamide was actually included in the synthesis of the LDH material, thereby inhibiting layer stacking and allowing only in-plane sheet growth as firstly reported by Sun and co-workers. ${ }^{[13 a]}$ Using this approach, monolayer NiFe-LDH with a lateral size of $30 \mathrm{~nm}$ and thickness of $\sim 1.0 \mathrm{~nm}$ could be successfully obtained in 10 min (denoted as LDH-monolayer, Figure 1A, B, Figure S1, S2) with the lattice spacing (0.15 nm) corresponding to the (110) planes of the LDH structure (Figure 1C). Ultrasonication of the LDH monolayer precursor in formamide caused fracturing of the nanosheets, thereby affording LDH-UF. Figure 1D and E show LDH-UF with a mean size of 2.3 $\mathrm{nm}$. A lattice fringe spacing of $0.15 \mathrm{~nm}$ also corresponds to the (110) planes of the LDH structure (Figure 1F), which was verified by performing a fast Fourier transform (FFT) on the high-resolution transmission electron microscopy (HRTEM) image (Figure 1G). The molar ratio of $\mathrm{Ni}: \mathrm{Fe}$ was identified as $2.90: 1: 00$, nearly the same as LDH- 
monolayer $(\mathrm{Ni}: \mathrm{Fe}=2.92: 1: 00)$. A structural model for NiFe-LDH-UF is shown in Figure S3, with an approximately nominal formula of $\left[\mathrm{Ni}_{42} \mathrm{Fe}_{14}(\mathrm{OH})_{112}\right]^{14+}$. Atomic force microscopy (AFM) was used to determine the thickness of the LDH-UF sample, a measured thickness of $\sim 0.6 \mathrm{~nm}$ is consistent with a single LDH monolayer (Figure 1H, I). The Tyndall effect was observed when an ethanolic suspension of LDH-UF was irradiated with a visible laser beam (Figure 1J), this provides evidence for the colloidal nature and the excellent dispersibility of the ultrafine LDH nanosheets.

On account of their monolayer thickness $(\sim 0.6 \mathrm{~nm})$ and small lateral size $(\sim 2.3 \mathrm{~nm})$, the LDH-UF were expected to contain an abundance of defects (such as oxygen or cation vacancies and low coordination metal ions located around the edge) as well as significant structural disorder, all of which could be expected to significantly alter the typical LDH electronic structure. X-ray absorption near-edge structure (XANES) and EXAFS were used to explore the effect of size reduction on LDH structure. Figure $2 \mathrm{~A}$ shows Ni K-edge XANES spectra for the LDH-UF and the monolayer LDH nanosheet precursor. Both showed similar spectra, with a slight difference seen above $8380 \mathrm{eV}$ for the ultrafine nanosheets suggesting a change in the electronic structure of $\mathrm{Ni}$ species. The Ni K-space spectra of the LDH-UF showed fewer oscillations at high $k$ values implying a subtle difference in the coordination environment of $\mathrm{Ni}$ atoms (Figure 2B). Key information about the Ni coordination was found in the corresponding R space plot (Figure 2C) which provided information on the first shell (Ni-O) and second shell (Ni$\mathrm{Ni}$ or Ni-Fe), the average distance $(R)$ and the coordination number $(N)$ in each shell. Compared with the Ni-O shell in LDH-monolayer $(N \sim 5.69)$, the Ni-O shell for the LDH-UF had a lower intensity and a lower $N$ (5.4), indicating severe structural distortion caused by the higher abundance of oxygen vacancies ( $\left.V_{\mathrm{O}}\right)$ in LDH-UF (Table $\mathrm{S} 2)$. Furthermore, in the Ni-Ni/Fe shell, $N$ decreased from 5.36 for the monolayer LDH nanosheets to 4.70 for $\mathrm{LDH}-\mathrm{UF}$, indicating the existence of $\mathrm{Ni}$ and $\mathrm{Fe}$ vacancies $\left(V_{\mathrm{Ni}}\right.$ and $\left.V_{\mathrm{Fe}}\right)$. The Debye-Waller factor $\left(\sigma^{2}\right)$ provides further evidence for severe structural distortion in the LDH-UF. The larger Debye-Waller factor for the Ni-O shell $(0.0059$ $\AA^{2}$ ) and $\mathrm{Ni}-\mathrm{Ni} / \mathrm{Fe}$ shell $\left(0.0066 \AA^{2}\right)$ for the LDH-UF, compared to the corresponding 
values for the LDH-monolayer (0.0047 and $0.0055 \AA^{2}$, respectively), suggest a severely distorted octahedral $\mathrm{Ni}-\mathrm{O}$ and $\mathrm{Ni}-\mathrm{Ni} / \mathrm{Fe}$ environments in the ultrafine nanosheets. Similarly at the Fe K-edge, the lower intensity of the signal for first Fe-O shell and second Fe-Ni shell of LDH-UF, compared with that of the LDH-monolayer (Figure 2DF, Table S3), implies more extensive defects and structural distortion to the LDH structure. The EXAFS data of Figure 2 conclusively demonstrate that as the lateral size of the monolayer $\mathrm{LDH}$ precursor was reduced to form the ultrafine nanosheets, both $V_{\mathrm{O}}$, $V_{\mathrm{Ni}}$ and $V_{\mathrm{Fe}}$ were introduced, leading to severe distortions in the $\mathrm{LDH}$ structure and metal cation coordination, mainly due to the severely increased edges sites of ultrafine nanosheets during synthesis. Recently, Wang and co-workers reported the development of multivacancies (metal and $\mathrm{O}$ vacancies) during the plasma exfoliation of bulk LDH into ultrathin nanosheets, supporting the findings of the current study. ${ }^{[11]}$ The presence of these defects and the structural distortion in the LDH-UF was expected to dramatically alter the $\mathrm{LDH}$ electronic structure and catalytic properties, as was confirmed by experiments and DFT calculations below.

We evaluated the OER performance of the NiFe-containing LDH-UF (lateral size $2.3 \mathrm{~nm}$ ) against monolayer NiFe-LDH (lateral size $\sim 30 \mathrm{~nm}$ ) and NiFe-LDH bulk (denoted as LDH-Bulk, lateral size $\sim 600 \mathrm{~nm}$ and thickness $\sim 20 \mathrm{~nm}$, Figure S4). Results are shown in Figure 3A. At a current density of $10 \mathrm{~mA} \mathrm{~cm}{ }^{-2}$, the LDH-UF, LDHmonolayer and LDH-Bulk displayed OER overpotentials $(\eta)$ of $0.254,0.280$ and 0.320 $\mathrm{V}$, respectively. Further, the current density of the LDH-UF was $\sim 2$ and $\sim 10$ times higher than that of LDH-monolayer and LDH-Bulk, respectively. The Tafel slope determined for LDH-UF was $\sim 32 \mathrm{mV} / \mathrm{dec}$ (Figure 3B), much smaller than that determined for LDH-monolayer $(42 \mathrm{mV} / \mathrm{dec})$ and LDH-bulk $(48 \mathrm{mV} / \mathrm{dec})$. These results confirm a superior OER performance for LDH-UF compared with previously tested LDH materials. To pinpoint the origin of the superior OER performance of the LDH-UF, electrochemical impedance spectroscopy (EIS) was used to explore the charge transfer properties of each LDH material. As shown in Figure 3C, the LDH-UF showed a much smaller semicircle in the Nyquist plot compared to LDH-monolayer or LDH-Bulk, 
indicating a significantly lower charge-transfer resistance. The high concentration of multivacancies in the ultrafine nanosheets, leading to the excellent conductivity, is thus very important to the high OER activity of the LDH-UF. ${ }^{[26]}$ Moreover, LDH-UF exhibited nearly a $100 \%$ Faradaic yield, indicating the $\mathrm{O}_{2}$ evolution was driven by LDH-UF electrocatalysts (Figure S5). The OER data indicates that LDH-UF show superior activity to most reported NiFe-based electrocatalysts for OER (Table S4). Figure 3D revealed that LDH-US maintained stable electrocatalytic activity over a 12 $\mathrm{h}$ period of OER testing.

Decreasing the LDH particle size is expected to increase the available surface area, although the electrochemical active surface area (ECSA) of the LDH-UF was $~ 10 \%$ higher than the LDH-monolayer (Figure S6), it was found that the current density of the LDH-UF was twice as high as that of the LDH-monolayer (Figure 3A), we can make a conclusion that factors other than just active surface area contribute to the high OER performance of the LDH-UF. Electronic structure and its influence on electrical conductivity clearly play a more significant role. ${ }^{[27]}$

In order to understand the impact of multivacancies $\left(V_{\mathrm{O}}, V_{\mathrm{Ni}}, V_{\mathrm{Fe}}\right)$ on the electronic structure of the NiFe-LDH ultrafine nanosheets, density functional theory (DFT) calculations performed. Structural models used for the calculations included monolayer $\mathrm{LDH}$, monolayer LDH doped with multivacancies $\left(V_{\mathrm{O}}, V_{\mathrm{Ni}}, V_{\mathrm{Fe}}\right)$ to represent the $\mathrm{LDH}-$ UF, and multilayered LDH (more than 3 layers). As shown in Figure 4A, B and Figure S7, multilayered NiFe-LDH without defects had a band gap of $\sim 1.4 \mathrm{eV}$. On reducing the LDH thickness to one monolayer, the band gap narrowed due to an increase in the Fe DOS near the Fermi level (Figure 4C, D and Figure S8). On introducing multivacancies $\left(V_{\mathrm{O}}, V_{\mathrm{Ni}}, V_{\mathrm{Fe}}\right)$ into monolayer $\mathrm{LDH}$, the DOS revealed no obvious gap around Fermi level (Figure 4E, F and Figure S9), indicating that the defects conferred semi-metal like character to the LD-UF. This result is in accordance with the electrochemical tests in Figure 3, which showed that the electrical conductivity and charge transfer properties of the LDH-UF were far superior to those of the monolayer LDH nanosheets. 
Finally, to demonstrate the wide applicability of the approach introduced here for the synthesis of NiFe-containing LDH-UF, we also synthesized ultrafine monolayer $\mathrm{ZnAl}-\mathrm{LDH}$ and monolayer NiCo-LDH nanosheets with lateral sizes of $\sim 3.0 \mathrm{~nm}$ and $\sim 2.8 \mathrm{~nm}$, respectively (Figure S10, S11).

In summary, the first successful synthesis of ultrafine sub-3 nm LDH nanosheet is reported, These materials display improved OER performance compared with the equivalent monolayer LDH. The synthesis method, is based on ultrasonication of monolayer LDH nanosheets with large lateral dimensions, the method is versatile and yields ultrafine LDH nanosheets in high yields with narrow size distributions. Reducing the lateral dimensions of monolayer LDH to less than $3 \mathrm{~nm}$ creates multivacancies and induces severe structural distortions, which collectively give the ultrafine nanosheets semi metal character and greatly improved OER activity compared with the monolayer nanosheets of lateral size $\sim 30 \mathrm{~nm}$. In conclusion, the work describes a facile route for the large production of ultrafine LDH nanosheets, paving the way for future widespread application in catalysis, energy applications, electronics, sensors and bio-imaging.

\section{Supporting Information}

Supporting Information is available from the Wiley Online Library or from the author.

\section{Acknowledgements}

The authors are grateful for the financial support from the National Key Projects for Fundamental Research and Development of China (2016YFB0600901, 2017YFA0206904, 2017YFA0206900), the Ministry of Science and Technology of China (2014CB239402, 2013CB834505), the Strategic Priority Research Program of the Chinese Academy of Sciences (XDB17000000), the National Natural Science Foundation of China $(51772305,51572270$, U1662118, 21401206, 21401207), the Young Elite Scientist Sponsorship Program by CAST (YESS) and the Youth Innovation Promotion Association of the CAS. The XAFS experiments were conducted in 1W1B beamline of Beijing Synchrotron Radiation Facility (BSRF). 
Received: ((will be filled in by the editorial staff))

Revised: ((will be filled in by the editorial staff))

Published online: ((will be filled in by the editorial staff))

\section{References}

[1] B. M. Hunter, H. B. Gray, A. M. Müller, Chem. Rev. 2016, 116, 14120.

[2] a) Q. Wang, D. O'Hare, Chem. Rev. 2012, 112, 4124; b) R. Ma, T. Sasaki, Acc. Chem. Res. 2015, 48, 136; c) G. Fan, F. Li, D. G. Evans, X. Duan, Chem. Soc. Rev. 2014, 43, 7040; d) S. He, Z. An, M. Wei, D. G. Evans, X. Duan, Chem. Commun. 2013, 49, 5912; e) M. Shao, R. Zhang, Z. Li, M. Wei, D. G. Evans, X. Duan, Chem. Commun. 2015, 51, 15880.

[3] a) M. Gong, Y. G. Li, H. L. Wang, Y. Y. Liang, J. Z. Wu, J. G. Zhou, J. Wang, T. Regier, F. Wei, H. J. Dai, J. Am. Chem. Soc. 2013, 135, 8452; b) M. R. Gao, W. C. Sheng, Z. B. Zhuang, Q. R. Fang, S. Gu, J. Jiang, Y. S. Yan, J. Am. Chem. Soc. 2014, 136, 7077; c) S.-M. Xu, H. Yan, M. Wei, J. Phys. Chem. C 2017, 121, 2683; d) F. Dionigi, P. Strasser, Adv. Energy Mater. 2016, 6, 1600621.

[4] F. Song, X. Hu, Nat. Commun. 2014, 5, 4477.

[5] F. Song, X. Hu, J. Am. Chem. Soc. 2014, 136, 16481.

[6] H. Liang, F. Meng, M. Caban-Acevedo, L. Li, A. Forticaux, L. Xiu, Z. Wang, S. Jin, Nano Lett. 2015, 15, 1421.

[7] K. Fan, H. Chen, Y. Ji, H. Huang, P. M. Claesson, Q. Daniel, B. Philippe, H. Rensmo, F. Li, Y. Luo, L. Sun, Nat. Commun. 2016, 7, 11981.

[8] K. Fan, Y. Ji, H. Zou, J. Zhang, B. Zhu, H. Chen, Q. Daniel, Y. Luo, J. Yu, L. Sun, Angew. Chem. Int. Ed. 2017, 56, 3289.

[9] X. Zhang, H. Cheng, H. Zhang, Adv. Mater., DOI: 10.1002/adma.201701704.

[10] Z. P. Liu, R. Z. Ma, M. Osada, N. Iyi, Y. Ebina, K. Takada, T. Sasaki, J. Am. Chem. Soc. 2006, 128, 4872.

[11] a) R. Liu, Y. Wang, D. Liu, Y. Zou, S. Wang, Adv. Mater. 2017, 29, 1701546; b) Y. Wang, Y. Zhang, Z. Liu, C. Xie, S. Feng, D. Liu, M. Shao, S. Wang, Angew. 


\section{WILEY-VCH}

Chem. Int. Ed. 2017, 56, 5867.

[12] G. Hu, D. O'Hare, J. Am. Chem. Soc. 2005, 127, 17808.

[13] a) J. Yu, B. R. Martin, A. Clearfield, Z. Luo, L. Sun, Nanoscale 2015, 7, 9448;

b) J. Yu, J. Liu, A. Clearfield, J. E. Sims, M. T. Speiegle, S. L. Suib, L. Sun, Inorg. Chem. 2016, 55, 12036.

[14] a) Y. Kuroda, Y. Miyamoto, M. Hibino, K. Yamaguchi, N. Mizuno, Chem. Mater. 2013, 25, 2291; b) N. Tarutani, Y. Tokudome, M. Jobbágy, F. A. Viva, G. J. A. A. Soler-Illia, M. Takahashi, Chem. Mater. 2016, 28, 5606; c) H. Li, T.-N. Tran, B.-J. Lee, C. Zhang, J.-D. Park, T.-H. Kang, J.-S. Yu, ACS Appl. Mater. Interfaces 2017, 9, 20294.

[15] a) J. Yu, Q. Wang, D. O'Hare, L. Sun, Chem. Soc. Rev. 2017, 46, 5950; b) G. Hu, N. Wang, D. O'Hare, J. Davis, Chem. Commun. 2006, 287.

[16] Y. Tokudome, T. Morimoto, N. Tarutani, P. D. Vaz, C. D. Nunes, V. Prevot, G. B. G. Stenning, M. Takahashi, ACS Nano 2016, 10, 5550.

[17] a) H.-J. Peng, Z.-W. Zhang, J.-Q. Huang, G. Zhang, J. Xie, W.-T. Xu, J.-L. Shi, X. Chen, X.-B. Cheng, Q. Zhang, Adv. Mater. 2016, 28, 9551; b) M. Lan, G. Fan, L. Yang, F. Li, Ind. Eng. Chem. Res. 2014, 53, 12943.

[18] C. Tan, X. Cao, X.-J. Wu, Q. He, J. Yang, X. Zhang, J. Chen, W. Zhao, S. Han, G.-H. Nam, M. Sindoro, H. Zhang, Chem. Rev. 2017, 117, 6225.

[19] a) R. Liu, D. Wu, X. Feng, K. Muellen, J. Am. Chem. Soc. 2011, 133, 15221; b) D. Pan, J. Zhang, Z. Li, M. Wu, Adv. Mater. 2010, 22, 734.

[20] a) H. J. Yu, Y. F. Zhao, C. Zhou, L. Shang, Y. Peng, Y. H. Cao, L. Z. Wu, C. H. Tung, T. R. Zhang, J. Mater. Chem. A 2014, 2, 3344; b) S. Zhu, Q. Meng, L. Wang, J. Zhang, Y. Song, H. Jin, K. Zhang, H. Sun, H. Wang, B. Yang, Angew. Chem. Int. Ed. 2013, 52, 3953.

[21] a) Z. Sun, H. Xie, S. Tang, X.-F. Yu, Z. Guo, J. Shao, H. Zhang, H. Huang, H. Wang, P. K. Chu, Angew. Chem. Int. Ed. 2015, 54, 11526; b) X. Zhang, H. Xie, Z. Liu, C. Tan, Z. Luo, H. Li, J. Lin, L. Sun, W. Chen, Z. Xu, L. Xie, W. Huang, H. Zhang, Angew. Chem. Int. Ed. 2015, 54, 3653. 


\section{WILEY-VCH}

[22] a) M. Honda, Y. Oaki, H. Imai, Phys. Chem. Chem. Phys. 2015, 17, 32498; b) X. Zhang, Z. Lai, Z. Liu, C. Tan, Y. Huang, B. Li, M. Zhao, L. Xie, W. Huang, H. Zhang, Angew. Chem. Int. Ed. 2015, 54, 5425; c) H. D. Ha, D. J. Han, J. S. Choi, M. Park, T. S. Seo, Small 2014, 10, 3858; d) X. B. Li, Z. J. Li, Y. J. Gao, Q. Y. Meng, S. Yu, R. G. Weiss, C. H. Tung, L. Z. Wu, Angew. Chem. Int. Ed. 2014, 53, 2085.

[23] J. Liu, Y. Liu, N. Liu, Y. Han, X. Zhang, H. Huang, Y. Lifshitz, S.-T. Lee, J. Zhong, Z. Kang, Science 2015, 347, 970.

[24] K. Nakamura, Y. Oaki, H. Imai, J. Am. Chem. Soc. 2013, 135, 4501.

[25] X. Wang, G. Sun, N. Li, P. Chen, Chem. Soc. Rev. 2016, 45, 2239.

[26] Y. Liu, H. Cheng, M. Lyu, S. Fan, Q. Liu, W. Zhang, Y. Zhi, C. Wang, C. Xiao, S. Wei, B. Ye, Y. Xie, J. Am. Chem. Soc. 2014, 136, 15670.

[27] Y. Sun, S. Gao, F. Lei, C. Xiao, Y. Xie, Acc Chem Res 2015, 48, 3. 


\section{WILEY-VCH}

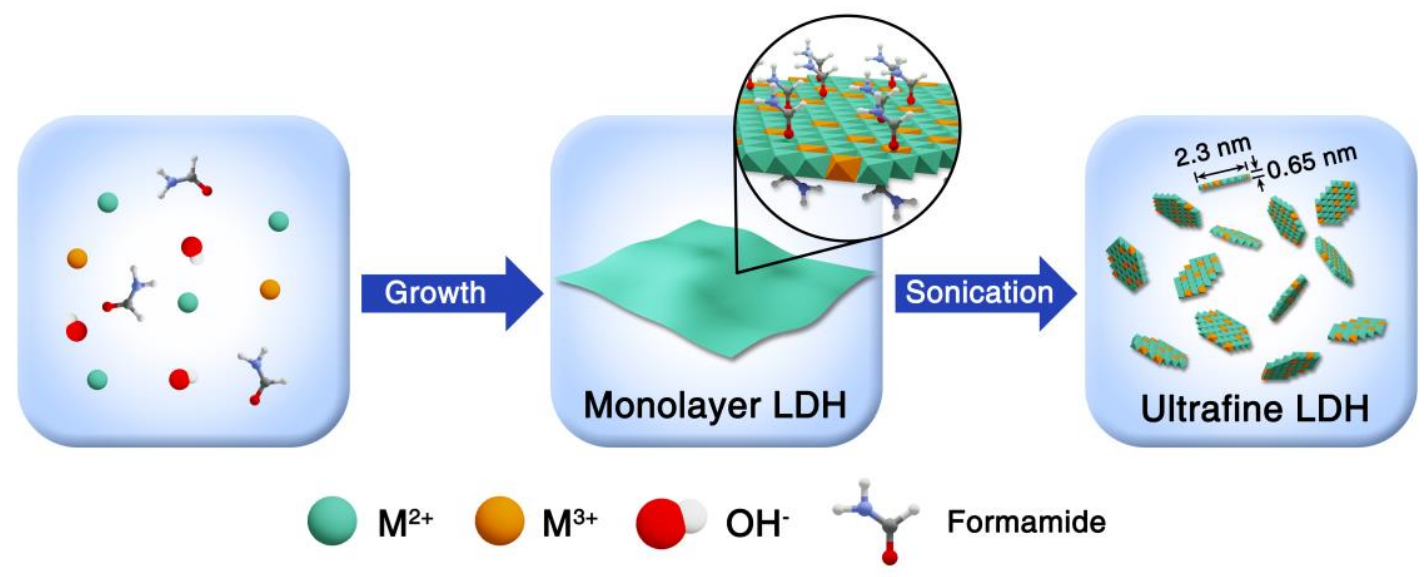

Scheme 1. Synthetic strategy used for the synthesis of ultrafine monolayer LDH nanosheets. 


\section{WILEY-VCH}
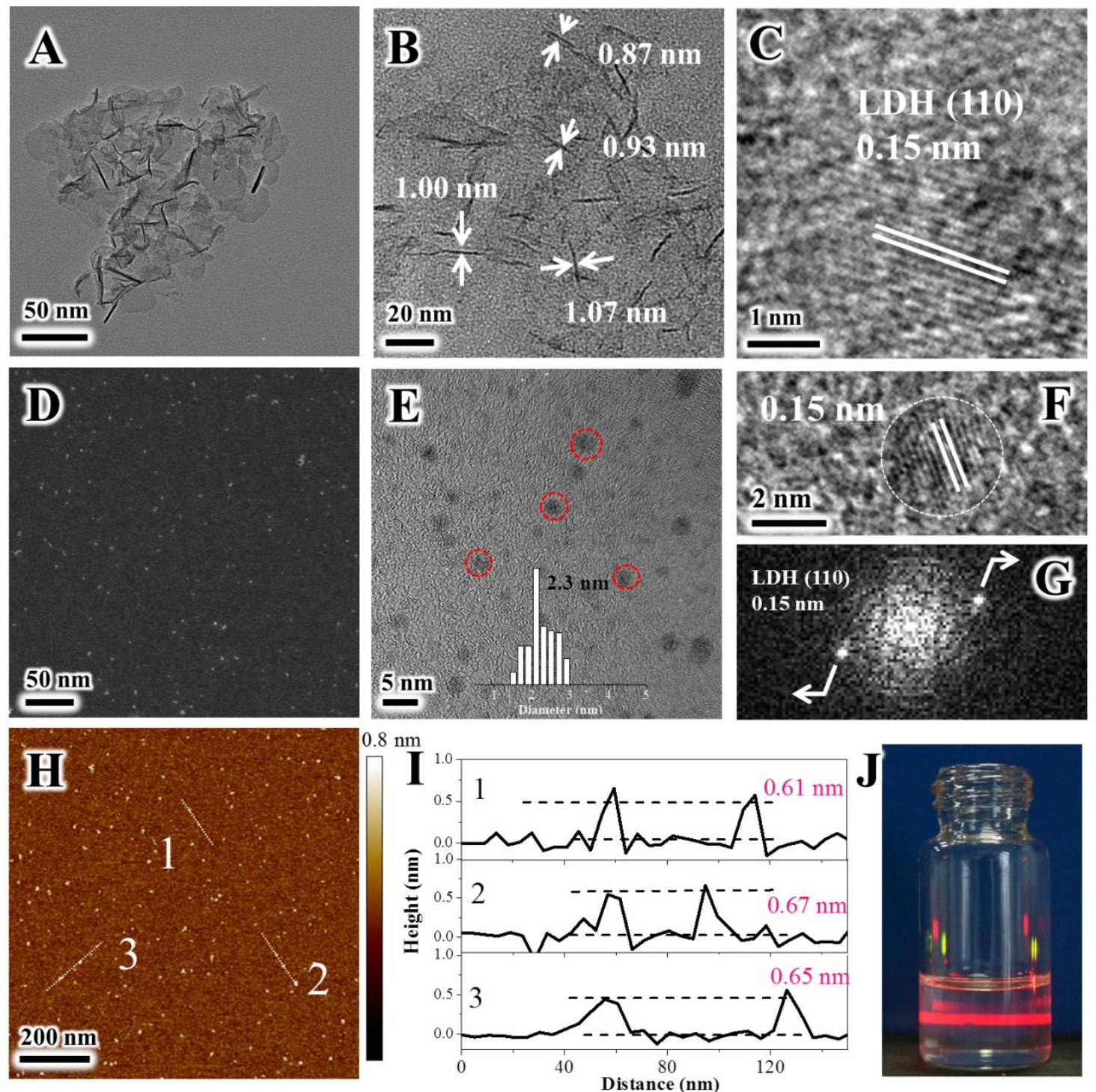

Figure 1. A, B) TEM and C) HRTEM images of the LDH-monolayer. D, E) High-angle annular dark-field TEM (HADDF-TEM) and HRTEM of LDH-UF, respectively. F) HRTEM image of one LDH-UF and G) the corresponding FFT pattern. H) AFM image and I) height profiles of LDH-UF. The numbers 1 to 3 in (I) correspond to the numbers 1 to 3 in $(\mathrm{H})$; J) A dispersion of the ultrafine $\mathrm{LDH}$ nanosheets in ethanol displaying Tyndall effect. 

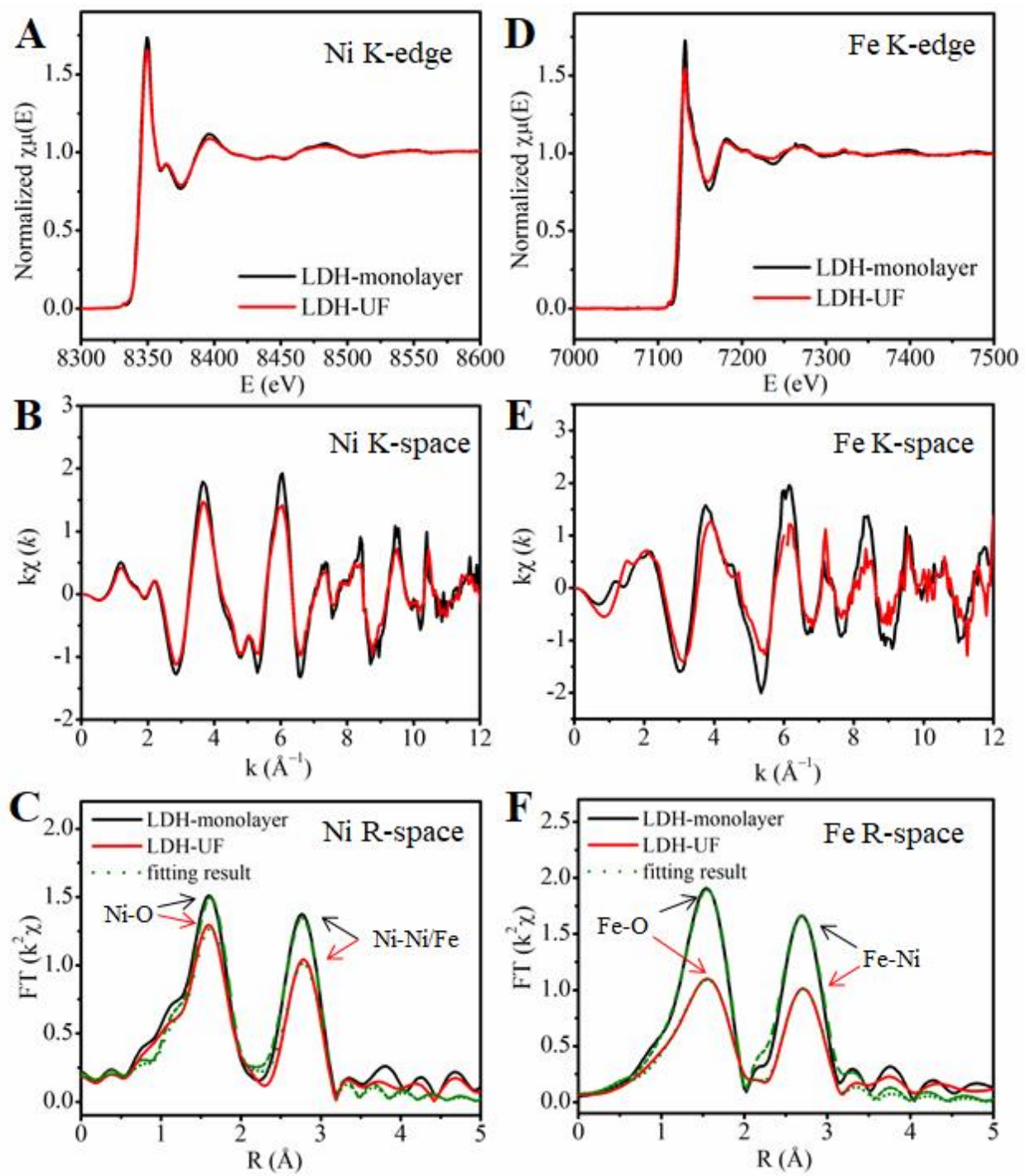

Figure 2. A) Ni K-edge XANES spectra, B) Ni K-edge EXAFS oscillation functions $k^{2} \chi(k)$ and C) magnitude of $k^{2}$-weighted FT of the Ni K-edge EXAFS spectra; D) Fe K-edge XANES spectra, E) Fe K-edge EXAFS oscillation functions $k^{2} \chi(k)$ and F) magnitude of $k^{2}$-weighted FT of the Fe K-edge EXAFS spectra for LDH-monolayer and LDH-UF, respectively. 

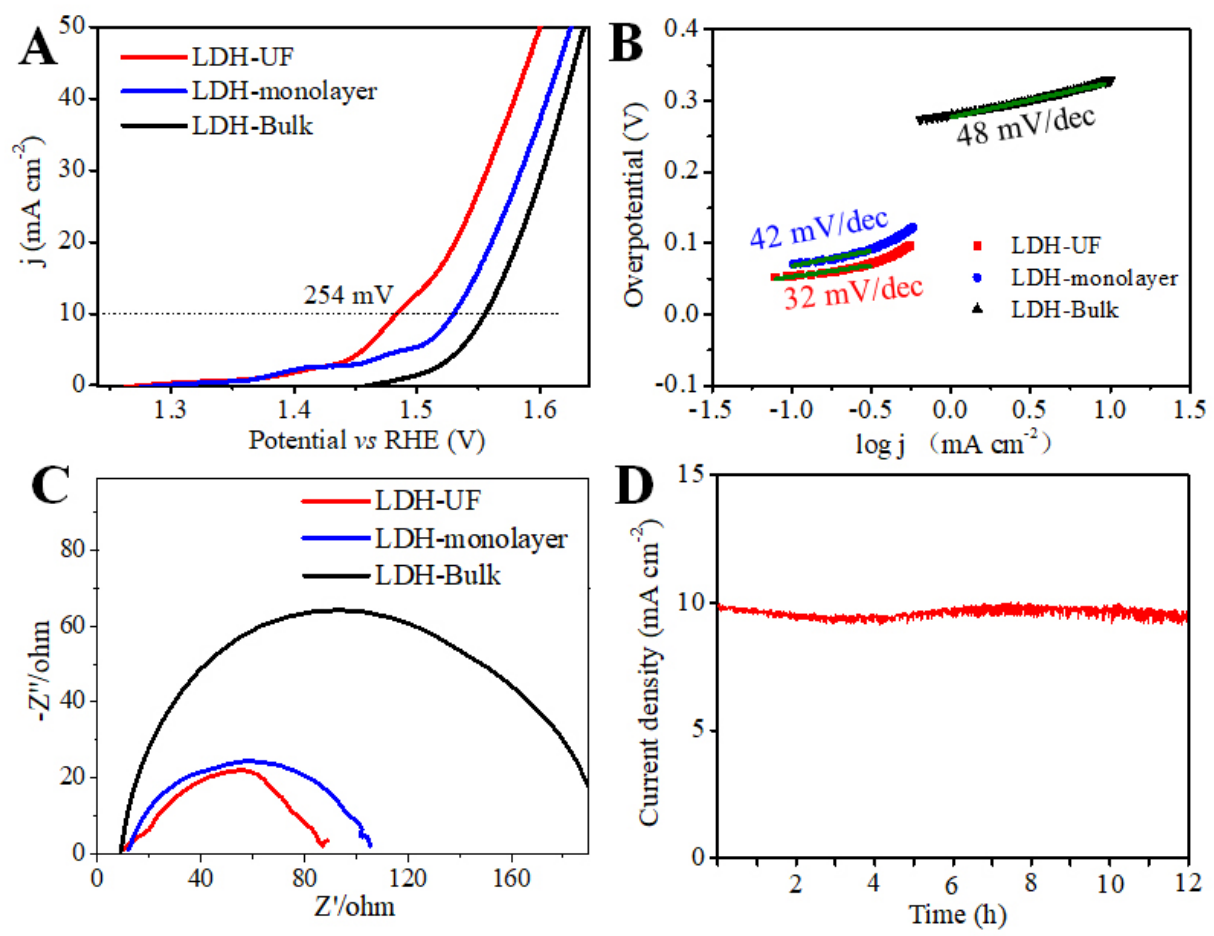

Figure 3. A) OER polarization curves for LDH-Bulk, LDH-monolayer and LDH-UF, respectively; sweep rate: $5 \mathrm{mV} \mathrm{s}^{-1}$; B) Tafel plots (RHE $v s$. log current) derived from A); C) Electrochemical impedance spectra for LDH-Bulk, LDH-monolayer and LDHUF electrodes; D) A current-time curve for water oxidation using LDH-UF at $\eta=254$ mV vs. RHE. 
WILEY-VCH
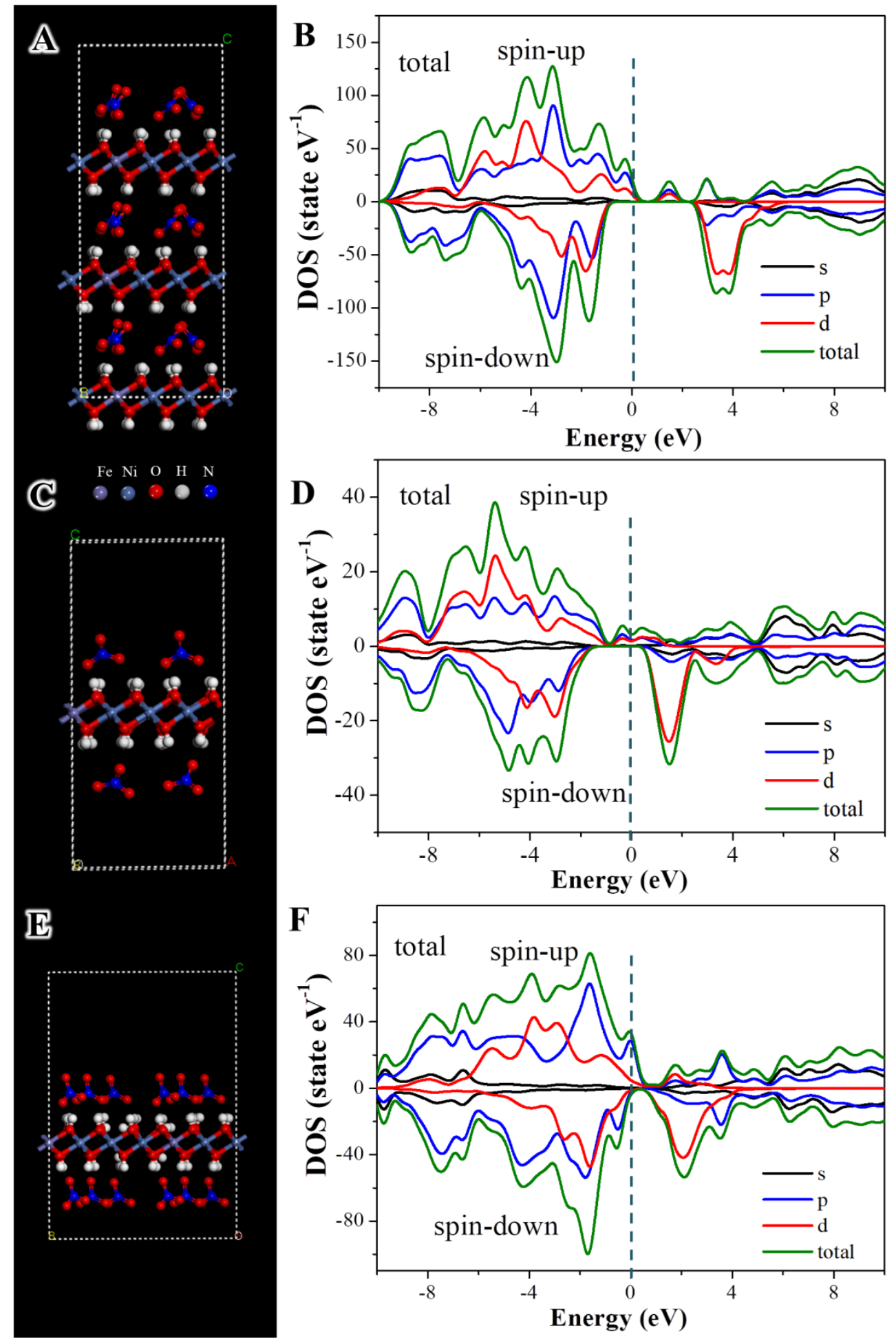

Figure 4. Schematic structure model and the corresponding calculated total DOS/PDOS plots for A, B) multilayer LDH; C, D) monolayer LDH and E, F) monolayer LDH-doped with multivacancies (The Fermi level is denoted by the vertical dashed line). 


\section{WILEY-VCH}

Ultrafine monolayer layered double hydroxide (LDH) nanosheets, with a mean lateral size of less than $3 \mathrm{~nm}$ were obtained by the ultrasonic treatment of monolayer LDH nanosheets. Abundant vacancies impart these ultrafine nanosheets with semimetallic character, superior charge transfer properties and electrocatalytic oxygen evolution performance relative to monolayer $\mathrm{LDH}$ nanosheets.

Keywords: layered double hydroxide; water oxidation, monolayer nanosheet; defect

Yufei Zhao, Xin Zhang, Xiaodan Jia, Geoffrey I.N. Waterhouse, Run Shi, Xuerui Zhang, Fei Zhan, Ye Tao, Li-Zhu Wu, Chen-Ho Tung, Dermot O'Hare and Tierui Zhang*

\section{Ultrafine NiFe-Layered Double Hydroxide Nanosheets for Water Oxidation}

TOC Figure:

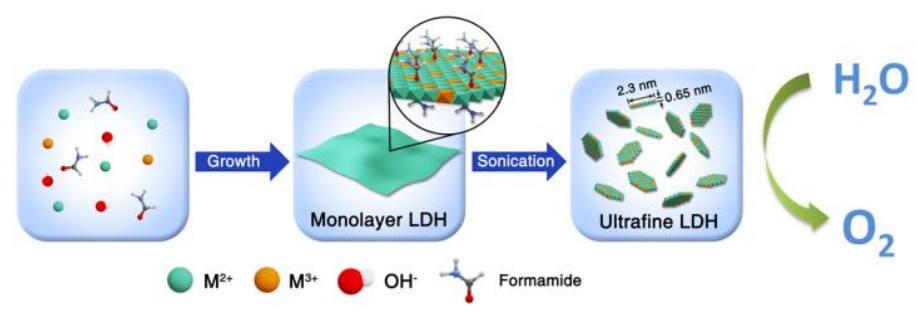

